\title{
Optimization of the Flow Path Efficiency in a Vacuum Cleaner Fan
}

\author{
In-Hyuk Son ${ }^{1}$ - Yoojeong Noh1,* - Eun-Ho Choi ${ }^{2}$ - Ju Yong Choi ${ }^{3}$ - Young Jin Ji ${ }^{4}$ - Kyungnae Lim ${ }^{4}$ \\ 1 Pusan National University, School of Mechanical Engineering, Republic of Korea \\ 2 Pusan National University, Pusan Educational Center for Computer Aided Machine Design, Republic of Korea \\ 3 Kyungsung University, Department of Mechatronics Engineering, Republic of Korea \\ ${ }^{4}$ LG Electronics, Motor R\&D Department, Republic of Korea
}

\begin{abstract}
As vacuum cleaners become smaller, the size of their main components must decrease correspondingly. Although compact vacuum cleaners have different flow characteristics in comparison to full-size vacuum cleaners, research on compact vacuum cleaners is limited. In this study, the fan motor unit for a compact vacuum cleaner, which is $30 \%$ smaller than a full-sized model, was evaluated by performing computational fluid dynamics analysis and design optimization. Since the flow path through the fan motor unit is the primary aspect determining the efficiency of the fan motor unit, the shape of the flow path is optimized. Through parametric studies, the dimensions of the diffuser underneath the fan, such as its thickness, fillet radius, and number of guide vanes, were selected as design variables. The design of the experiments was used to find the optimum design of the diffuser and guide vanes to maximize the flow path efficiency of the cleaner. The optimized model improved the flow path efficiency by $4.2 \%$ in comparison to the initial model.
\end{abstract}

Keywords: CFD analysis, design optimization, design of experiments, vacuum cleaner fan, flow path efficiency

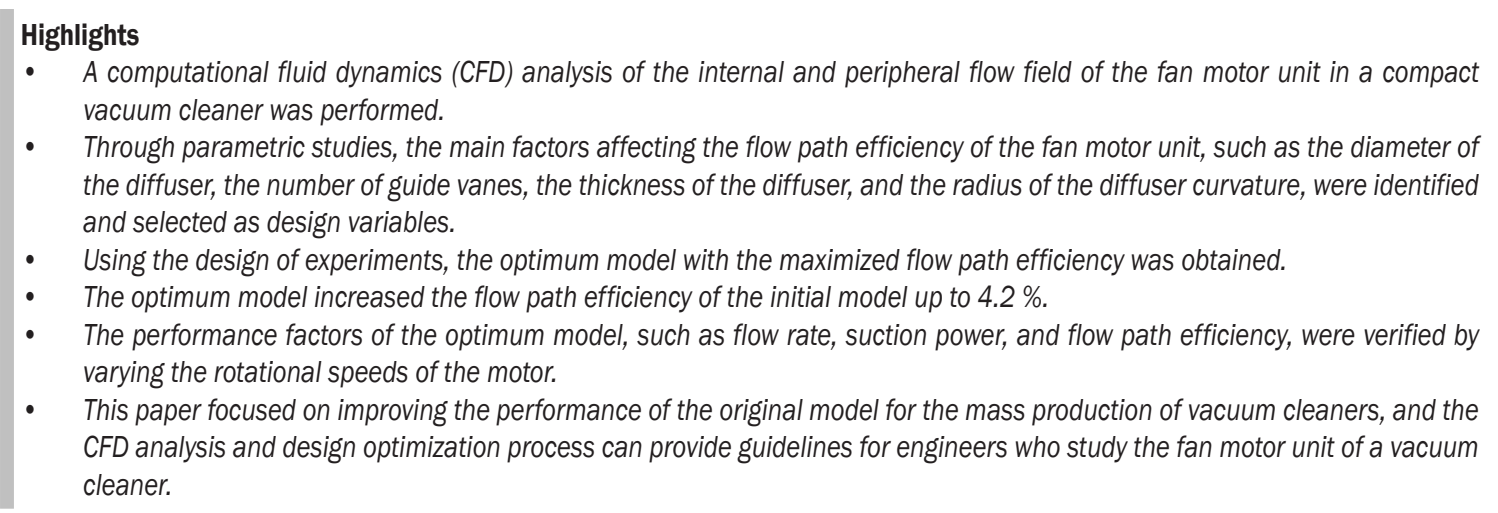

\section{INTRODUCTION}

Similar to washing machines, refrigerators, and televisions, vacuum cleaners have become an essential appliance in modern society. Many vacuum cleaners have been launched in the market; to meet the demands of consumers, their performance is continually being improved, and their sizes are becoming ever smaller. As vacuum cleaners decrease in size, the motor and fan, which are the main components, also need to be miniaturized. The full-size model is mainly an upright or a canister type, which has high suction power, but it is inconvenient to carry. Otherwise, a compact model is mainly a stick type or handheld type, which is cordless and lightweight; it has recently become popular. However, compact models have different characteristics compared to full-size models. For example, the motors of compact models must rotate more rapidly than those of full-size models, and the input torques and the diameters of the inlet ports of the cleaning orifices in compact models are smaller than those of full-size models. Therefore, the flow characteristics of compact models are quite different than those of full-size models.

The flow inside a turbo fan rotating at high speed is different from the flow through a general fan, due to the very low pressure at the inlet of the fan, where the low-pressure state gradually returns to atmospheric pressure as the air passes through the fan and diffuser. In this process, the flow inside the fan and diffuser becomes quite complex and unstable [1]. In fact, it is difficult to grasp the flow through each element in a fan motor unit; thus, only the overall performance and flow characteristics of a fan are obtained through experimental results for real applications. In addition, it is unclear how the internal components of a fan motor unit affect its performance. It is uncertain as to whether the analysis results for each 
independent element consider the interactive effects among elements; thus, they are often rather different in comparison to real experimental results [2]. Accordingly, it is necessary to predict the performance of a fan motor unit through accurate CFD analysis inside the unit; additionally, the shape of the internal structure must be designed by identifying the main parameters that affect its performance. However, the internal structure and the electromagnetic mechanism of the motor are complicated, and the internal space is very restricted, such that the design of the internal structure is practically difficult. Moreover, it is necessary to consider the safety of the users of the electronic product as well as the electrical and mechanical stability; thus, the design domain for improving the performance of the fan motor unit is very limited.

Fans are widely used in myriad mechanical products, so extensive studies on the analysis of the flow around a fan or design of the impeller shape exist. Kim et al. [3] and Kale et al. [4] analysed the flow field of a centrifugal fan for a vacuum cleaner to investigate the cause of motor noise and validated the analysis results through experiments. Rihtaršič et al. [5] investigated the flow through the centrifugal impeller of a vacuum cleaner unit, using experimental measurements and numerical simulations. Li [6] performed flow analysis on a fan for a centrifugal fan motor system with a ported diffuser and calculated the efficiencies according to the orifice diameter and the energy loss for each component of the fan motor system. Park et al. [7] performed CFD analysis on centrifugal fans for a vacuum cleaner and analysed the effect of the shape of the guide vanes on the suction performance. Lee [8] improved the efficiency of a centrifugal fan by varying the geometric dimensions of the impeller. Park [2] proposed a methodology for constructing a system level analysis of a fan motor assembly by combining characteristics of each component through torque matching of the fan and motor. Kim et al. [9] performed a multidisciplinary optimization of an axial flow fan to enhance the aerodynamic and aero-acoustic performance. However, these studies mostly dealt with flow analysis related to noise or vibration, or they only included analysis of the flow around the fan in the fan motor system of a full-size model. A previous study carried out a system level analysis of a fan motor assembly, but it did not optimize its design. Studies on the overall flow analysis and design optimization of the internal structure have not been performed for the fan motor system of a compact model.
In this study, the optimal design of a fan was performed via CFD analysis for a compact model whose size is reduced by $30 \%$ in comparison to the corresponding full-size model. The design parameters of the thickness, diameter, radius of curvature, and number of guide vanes were selected through parametric studies using the commercial optimization software tool, PIAnO [10]. Using this software, the flow path around the diffuser was designed to maximize the flow path efficiency through the design of experiment (DOE). ANSYS CFX was used for the CFD analysis, and the computational analysis was verified by comparing the initial model and the optimum [11] and [12].

\section{CFD ANALYSIS METHOD}

For CFD analysis, there are the $k-\varepsilon, k-\omega$, and shearstress transport (SST) turbulence models. The $k-\varepsilon$ model precisely predicts the flow outside the boundary layer, but it has difficulty predicting flow within the boundary layer [9] and [13]. Unlike the $k-\varepsilon$ model, the $k-\omega$ model is advantageous for analysing near-wall regions and accurately predicting the flow inside of the boundary layer [14] and [15]. The SST model is a turbulence model that connects the two preceding models with blending functions included in ANSYS CFX. It uses the $k-\varepsilon$ model in the fully developed region and automatically uses the $k-\omega$ model near the wall; thus, it predicts the flow separation due to the back-pressure gradient and accurately performs the turbulent flow analysis [13]. In this study, the CFD analysis was performed using the SST model.

Because the SST model uses the $k-\omega$ model near the wall and uses the $k-\varepsilon$ model far from the wall, the transformed equations for the SST $k-\omega$ and $k-\varepsilon$ model are given by [16] and [17]:

$$
\begin{gathered}
\frac{\partial(\rho k)}{\partial t}+\frac{\partial\left(\rho U_{i} k\right)}{\partial x_{i}}= \\
=\frac{\partial}{\partial x_{j}}\left[\left(\mu+\sigma_{k} \mu_{t}\right) \frac{\partial k}{\partial x_{j}}\right]+P_{k}-\beta^{*} \rho \omega k, \\
\frac{\partial(\rho \omega)}{\partial t}+\frac{\partial\left(\rho U_{i} \omega\right)}{\partial x_{i}}=\frac{\partial}{\partial x_{j}}\left[\left(\mu+\sigma_{\omega} \mu_{t}\right) \frac{\partial \omega}{\partial x_{j}}\right] \\
+2\left(1-F_{1}\right) \frac{\rho \sigma_{\omega 2}}{\omega} \frac{\partial k}{\partial x_{j}} \frac{\partial \omega}{\partial x_{j}}+\frac{\alpha}{v_{t}} P_{k}-\beta \rho \omega^{2},
\end{gathered}
$$

where $x_{i}$ and $x_{j}$ are Cartesian coordinates for $i$ and $j=1$ to $3, k$ is the kinetic energy, $\rho$ is the specific mass (i.e. density), $U$ is the velocity, $t$ is the time, $\omega$ is the turbulence frequency, $\mu_{t}$ is the turbulent eddy viscosity, 
$\mu$ is the molecular viscosity of the fluid, and $P_{k}$ is the shear production of turbulence, which is given by:

$$
P_{k}=\left(2 \mu_{t} S_{i j}-\frac{2}{3} \rho k \delta_{i j}\right) \frac{\partial U_{i}}{\partial x_{j}},
$$

where $S_{i j}$ represents the mean rate of deformation components, and $\delta_{i j}$ is the Kronecker delta function.

All constants used in the SST model are obtained by combining the corresponding constants of the two models using a blending function, $F_{1}$ : $\alpha=\alpha_{1} F_{1}+\alpha_{2}\left(1-F_{1}\right)$. The constants for this model are $\beta^{*}=0.09, \quad \beta_{1}=3 / 40, \quad \beta_{2}=0.0828, \quad \alpha_{1}=5 / 9, \quad \alpha_{2}=0.44$, $\sigma_{k 1}=0.85, \sigma_{k 2}=1, \sigma_{\omega 1}=0.5$, and $\sigma_{\omega 2}=0.856$. The subscript 1 is related to $k-\omega$ model, and the subscript 2 is connected with the $k-\varepsilon$ model. The blending function is defined by:

$$
F_{1}=\tanh \left\{\left\{\min \left[\max \left(\frac{\sqrt{k}}{\beta^{*} \omega y}, \frac{500 \mu}{y^{2} \omega}\right), \frac{4 \rho \sigma_{\omega 2} k}{C D_{k \omega} y^{2}}\right]\right\}^{4}\right\},
$$

where $C D_{k \omega}=\max \left(2 \rho \sigma_{\omega 2} \frac{1}{\omega} \frac{\partial k}{\partial x_{i}} \frac{\partial \omega}{\partial x_{i}}, 10^{-10}\right)$ and $y$ is the distance to the nearest wall.

The turbulent eddy viscosity is defined as:

$$
v_{t}=\frac{a_{1} k}{\max \left(a_{1} \omega, S F_{2}\right)},
$$

where $S$ is the invariant measure of the strain rate given by $\sqrt{2 S_{i j} S_{i j}}$ and $F_{2}$, which is the second blending function:

$$
F_{2}=\tanh \left[\left\{\max \left(\frac{2 \sqrt{k}}{\beta^{*} \omega y}, \frac{500 \mu}{y^{2} \omega}\right)\right\}^{2}\right] .
$$

Because this model requires the distance between the nodes and the nearest wall (WD), the wall scale equation is used:

$$
W D=\sqrt{|\nabla \phi|^{2}+2 \phi-|\nabla \phi|},
$$

where $\phi$ is the value of the wall scale, which is defined by the ANSYS user's guide according to the rotor type.

There are various numerical analysis methods for analysing the internal flow field of a turbo fluid machine rotating at high speed. Typically, the multiple reference frames (MRF) method is used for steadystate analysis, and the sliding mesh (SM) method is used for transient analysis [18]. In this study, CFD analysis is performed using ANSYS CFX for steadystate analysis via the MRF method, which can shorten the computational time. In addition, to apply the MRF method to CFX, it is necessary to set the interface between the rotating body and the fixed part, wherein the fan and the other parts are considered as the rotating body and fixed bodies, respectively [11]. To set the interface between the rotating body and the fixed part, there are "Frozen Rotor" and "Stage" in the menu of ANSYS CFX. The "Frozen Rotor" is the steady-state method that uses the rotating reference frame to save computational time by converting the transient flow in the steady-state. The "Stage" calculates the averaged results for the flow separation and vortex generated in the upstream and downstream, so that it is more accurate than the "Frozen Rotor" and generally used in high-speed turbo-machines. In this study, the "Stage" method was used.

\section{SIMULATION}

\subsection{Geometric Model}

The vacuum cleaner can be divided into the air inlet, fan, motor, and exhaust. In this model, a brushless DC (BLDC) motor was used. To evaluate the performance of the cleaner, it is necessary to analyse the entire flow in the fan motor unit, but the flow around the fan and its related components has the greatest influence on the performance. Therefore, this study aims to improve the flow path efficiency by reducing the flow loss around the fan, and the main analytical model is limited to the periphery of the fan.

Internal airflow is generated by the fan rotating at high speed, and the pressure difference between the inlet and the outlet forces the airflow to the exhaust portion. The diffuser located directly behind the fan directs the flow from the motor to the discharge portion. Flow losses are inevitably generated in these serial flows. To reduce these flow losses, i.e. increase the flow path efficiency, the baseline flow characteristics inside the fan motor unit must be determined.

Fig. 1 shows the main parts of the fan motor unit of the vacuum cleaner. The fan is connected to the inlet port and located on the upper part of the fanmotor unit, and the diffuser is located just below the fan. The fan and the diffuser are surrounded by an upper casing made of steel.

The motor, coils, shaft, and the body inside the fan motor unit are surrounded by a lower casing, wherein the shaft and coils are wound with several electrical devices underneath the diffuser. The diameters of the fan and diffuser are $34 \mathrm{~mm}$ and $47.2 \mathrm{~mm}$, respectively, and the total height is $56.7 \mathrm{~mm}$. 

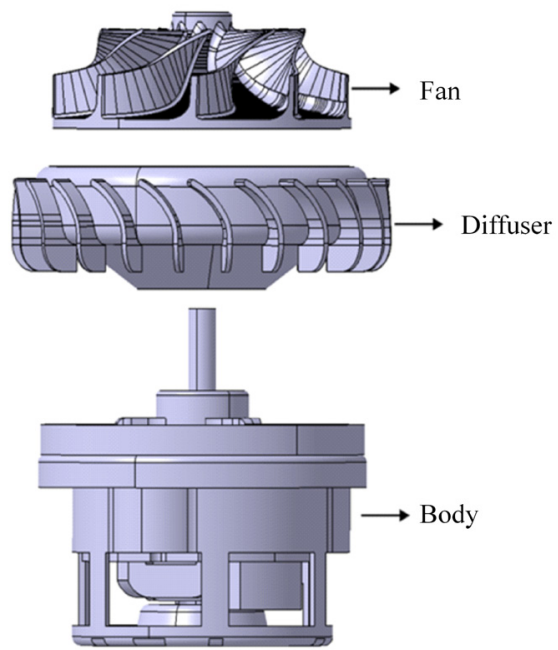

Fig. 1. Inner components of the motor unit for the compact vacuum cleaner

\subsection{Finite Element Model}

It is essential to observe the flow of the inlet, at which the air is drawn in, and the flow of the outlet, at which the air is exhausted. Thus, a finer mesh was used in those regions. Tetrahedron meshes with minimum and maximum dimensions of $0.1 \mathrm{~mm}$ and $10 \mathrm{~mm}$, respectively, were created using the "form new part" function in CFX, which shares the nodal points between each region during the meshing process for the rotating body (fan) and the non-rotating bodies (other parts). The mesh generation affects the analysis results according to the mesh dimensions. In the case of this model having contact regions between the rotating and fixed bodies, the meshes are generated using the "form new part" function because the analysis error can generally be reduced by sharing the node points. To generate the finite element model, a constant mesh was created by separating the impeller part, which is the rotation area, and the rest, which is the stationary part, and the turbulence model was a $k-\omega$ model more suitable for the nonlinear flow analysis.

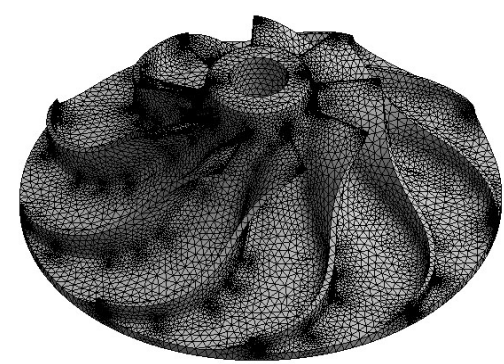

Fig. 2. FE model of the fan
Fig. 2 shows the finite element (FE) model of the rotating fan with 1.1 million meshes and 108,000 nodes, and Fig. 3 shows the FE model for the interfaces between rotary and stationary parts.

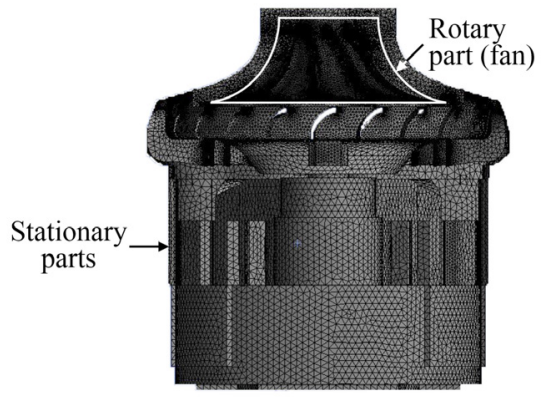

Fig. 3. FE model of rotary and stationary parts

Fig. 4 indicates the entire FE model of the fan motor unit, which consists of 3.8 million elements (including those of the fan). The diameters of the orifice region and vacuum region, which are modelled by cylinders, are $500 \mathrm{~mm}$, and their heights are $500 \mathrm{~mm}$. The ambient region is smaller than the vacuum or orifice region; its diameter is $400 \mathrm{~mm}$, and its height is $400 \mathrm{~mm}$. The dimensions of all regions are set to be identical to the testing conditions of the fan motor unit in the company.

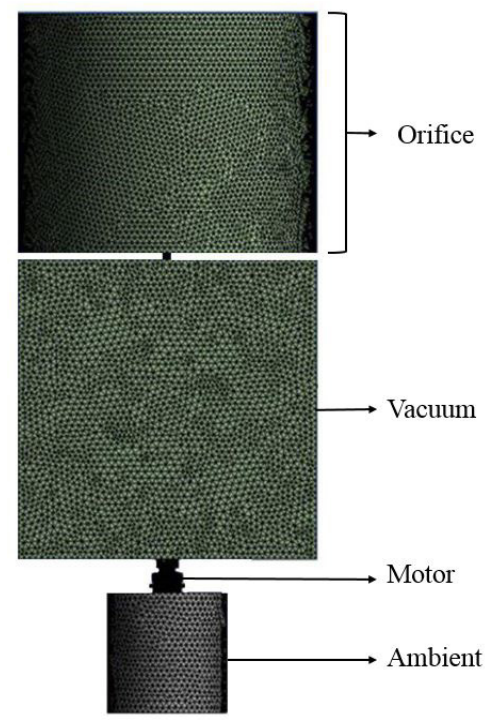

Fig. 4. Full FE models for the motor unit and its surrounding area

The inlet and outlet pressures were set to the same value to express the principle of the fluid machine, which generates the flow by rotating the internal fan when there is no pressure difference at the inlet and outlet. Fig. $5 \mathrm{c}$ shows the fan domain region, which is 
used to set the rotation speed and rotational direction of the fan. To provide the rotational effect to the rotating fan, the "Move element" function was applied in the CFX pre-processing step. This applies the rotational effect to the fan by rotating the surrounding airflow in the opposite direction without rotating the fan. Accordingly, for the original rotation speed of $90,000 \mathrm{rpm}$ in the clockwise direction, "Move element" uses a rotation speed of 90,000 rpm in the counter-clockwise direction, which is applied at the inlet and the wall of the rotating airflow region.

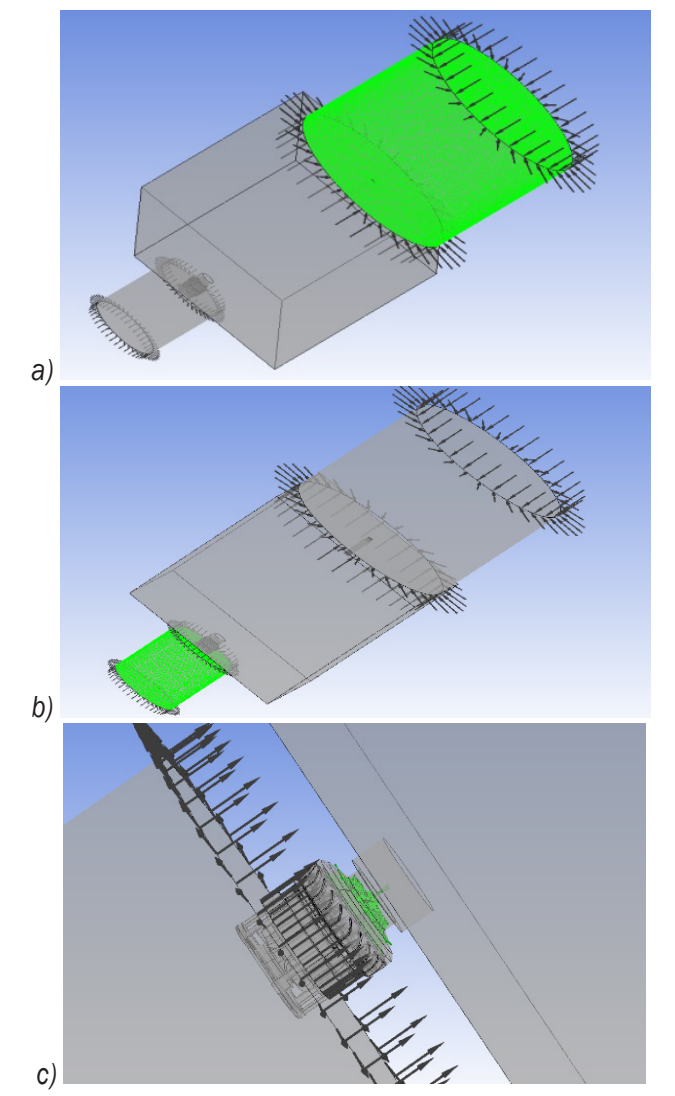

Fig. 5. Boundary conditions; a) inlet, b) outlet, and c) main body

Fig. 6 shows velocity streamlines of the airflow passing through all the flow paths from the inlet to the outlet. The air is drawn into the orifice, and the velocity of the air passing through the orifice reaches the maximum value. The velocity of the air gradually decreases and then passes through the inlet of the fan motor unit with high velocity, especially near the fan. It is finally discharged to the outside, i.e. the ambient region.

In the solver control stage, 1,000 repetitions were used after generating meshes. For the time scale factor, the default value of 1 was used, as decreasing the value increases both accuracy and computation time. The residual target of the mass conservation equation was set to $10^{-4}$, which is often used as a residual target. The computational time was shortened by using the platform message passing interface (MPI) local parallel, which connects central processing unit (CPU) cores in parallel instead of using serial cores, thereby accelerating the computation of the CFD analysis.

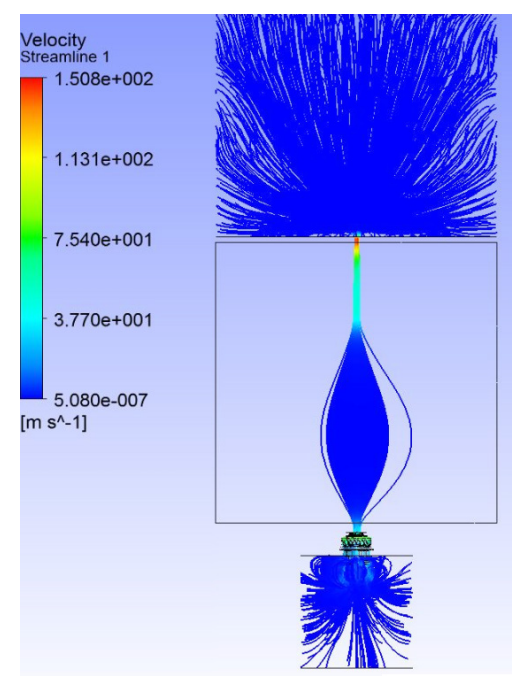

Fig. 6. CFD result of a full model

\section{DESIGN OPTIMIZATION}

\subsection{Parametric Study}

The shape of the impeller was already optimized by the company, and the motor has design restrictions due to the complicatedly wound coils around the motor. In addition, the shape of the lower body of the fan motor unit has little influence on the flow path efficiency since the velocity of the airflow decreases after passing through the guide vane. The diameter of the orifice helps to increase the air flow rate, but a motor with higher capacity and a larger motor casing needs to be considered to achieve high flow path efficiency, which could complicate the optimization of the flow efficiency in the vacuum cleaner fan. Therefore, the impeller, the flow path shape around the motor, the lower body of the fan motor unit, and the diameter of the orifice are excluded as design variables. However, the diffuser located underneath the impeller directly affects the performance of the fan motor unit, but its shape has yet to be considered as a design variable. Thus, in this study, the design variables related to the shape of the diffuser were selected through the parametric study using PIAnO. 
Because the diffuser possesses six design variables, which is a large number for the DOE, it is necessary to reduce the number of design variables [19]. To achieve this, Plackett-Burman design (PBD) was used; this design method ignores the high-order interactions between several design variables and prioritizes the main effects between pairs of design variables on the output performance. The design variables used in the analysis of the main factors are the diffuser diameter $\left(X_{1}\right)$, the number of guide vanes $\left(X_{2}\right)$, the diffuser thickness $\left(X_{3}\right)$, the curvature radius of the diffuser $\left(X_{4}\right)$, the curvature radius of the vane $\left(X_{5}\right)$, and the width of the vane $\left(X_{6}\right)$, as shown in Fig. 7.
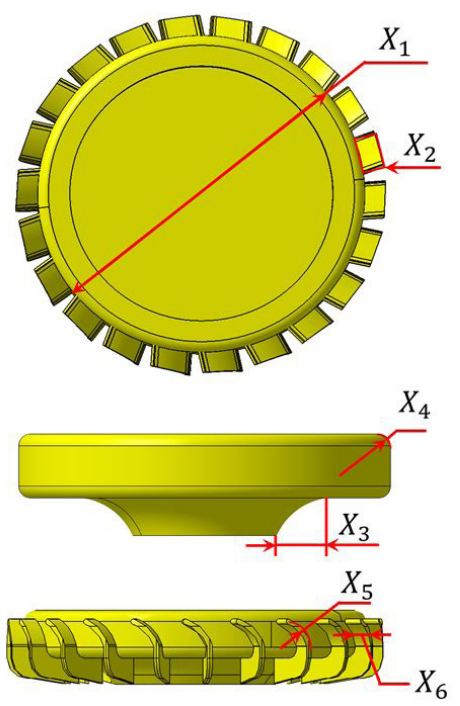

Fig. 7. Design variables for the parametric study

The lower bound (L/B) and upper bound (U/B) for each design variable are shown in Table 1 . The design bounds were determined considering design limitations because the fan and diffuser should be assembled with the existing casing, motor, and coils with fixed shapes and sizes.

Table 1. Design bounds for DOE (in $\mathrm{mm}$ )

\begin{tabular}{lcccccc}
\hline Intact & $X_{1}$ & $X_{2}$ & $X_{3}$ & $X_{4}$ & $X_{5}$ & $X_{6}$ \\
\hline L/B & 45.2 & 16 & 6.3 & 0 & 0.5 & 1.5 \\
\hline Middle & 47.2 & 19 & 7.3 & 1 & 1.0 & 2.5 \\
\hline U/B & 49.2 & 22 & 8.3 & 2 & 1.5 & 3.5 \\
\hline
\end{tabular}

As results of the parametric study, the four most essential design variables are the diameter of the diffuser $\left(X_{1}\right)$, the number of guide vanes $\left(X_{2}\right)$, the thickness of the diffuser $\left(X_{3}\right)$, and the radius of the diffuser curvature $\left(X_{4}\right)$, as shown in Fig. 8. As the air enters the impeller and leaves the diffuser, the airflow is abruptly changed, owing to the sharp change of the flow path. Thus, the obtained CFD results are rather different according to the corresponding lower and upper bounds of design variables $X_{1}$ through $X_{4}$.
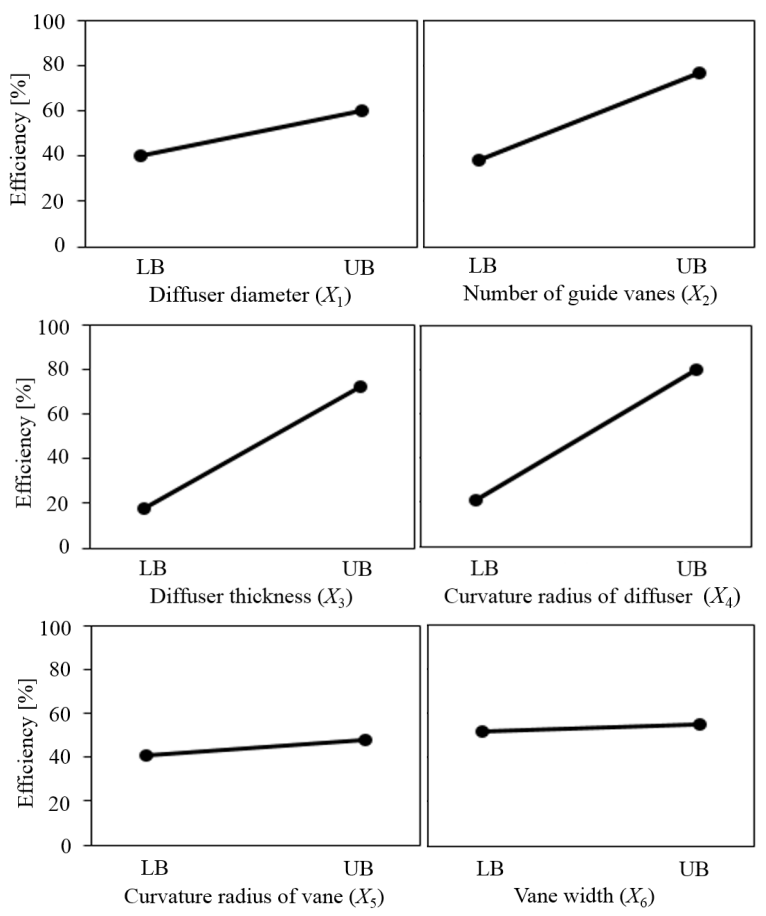

Fig. 8. Results of the parametric study for $X_{1}$ to $X_{6}$

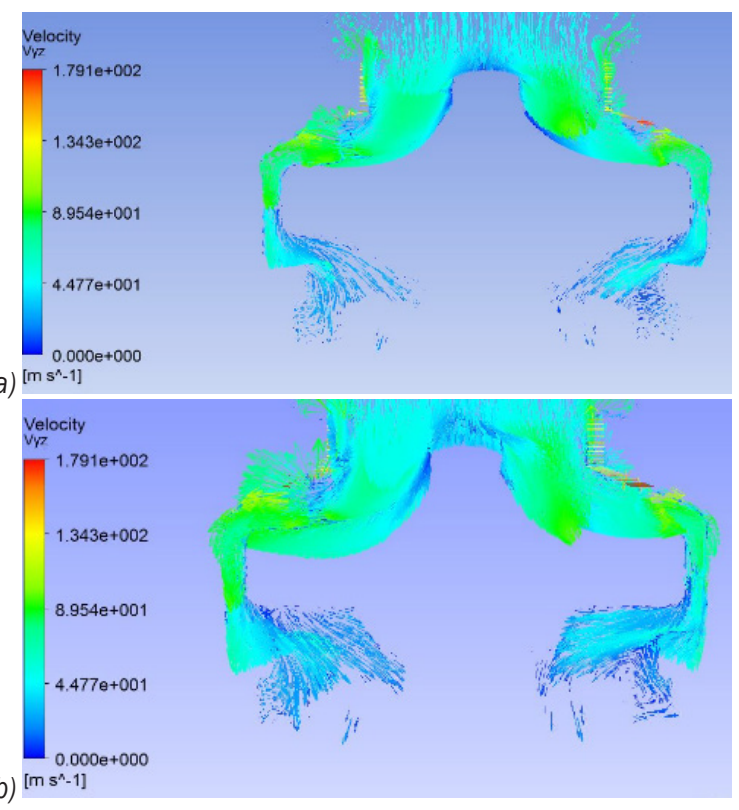

Fig. 9. CFD results for $X_{3}$; a) lower bound, and b) upper bound

For example, Fig. 9 shows the velocity vectors for the lower and upper bounds of $X_{3}$. As the curvature 
radius of the diffuser increases, the air leaving from the guide vane passes more smoothly through the lower part of the diffuser, and the flow path efficiency increases correspondingly.

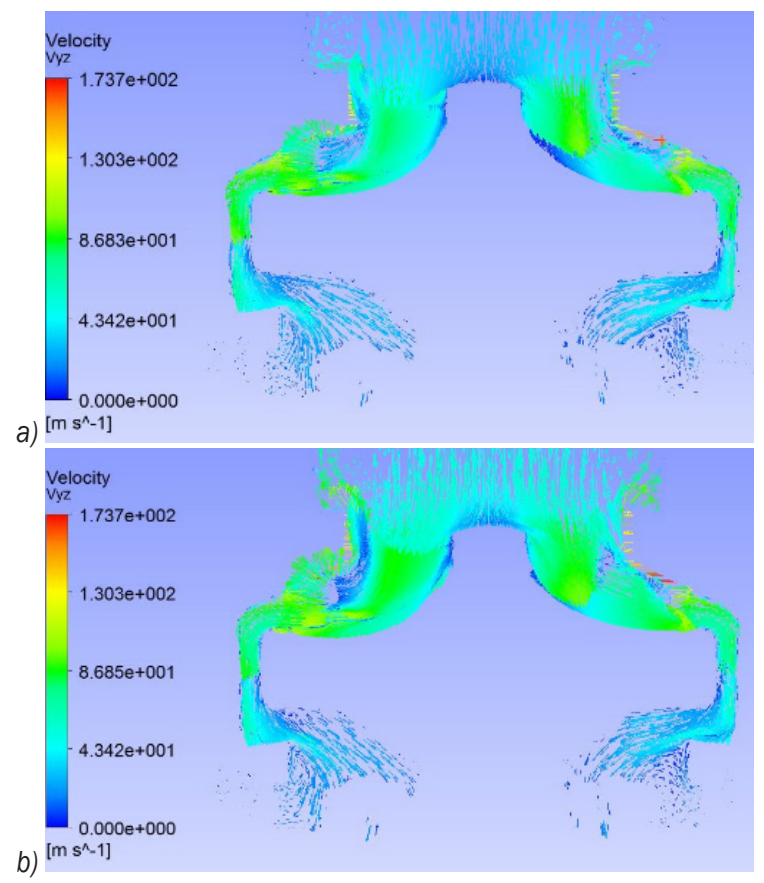

Fig. 10. CFD results for $X_{5}$; a) lower bound, and b) upper bound

In contrast, the guide vane only induces the air from the impeller to the diffuser, such that changes to the shape of the guide vane $\left(X_{5}\right.$ and $\left.X_{6}\right)$ have little effect on the flow efficiency compared to the other design variables, as shown in Figs. 9 and 10. Thus, only the four design variables that have much effect on the airflow $\left(X_{1}\right.$ through $\left.X_{4}\right)$ were considered as design variables for the design optimization.

\subsection{Design Optimization}

This section describes the methodology used to design the diffuser for maximizing the flow path efficiency. The objective function is the flow path efficiency, which is expressed as the ratio of the fluid power $P_{\text {air }}$ to the input shaft power $P_{\text {shaff. }}$. The fluid power $P_{\text {air }}$ is calculated as the multiplication of the average pressure of the entire surface of the vacuum region, $P_{s}$, and the flow rate, $Q$. The flow rate represents the flow rate of the airflow passing through the orifice. The input power of the shaft, $P_{\text {shaff }}$, is obtained from the product of the input torque $(T)$ and the rotation speed $(N)$ multiplied by $2 \pi / 60$ to transform from dimensions of "rpm" to "rad/s". The input power needs to be larger than the current output power, i.e. $350 \mathrm{~W}$.
The initial values of design variables and their lower and upper bounds are also the same as those in Table 1. To maximize the flow path efficiency, the pressure of the output flow should be maximized for a given input power, which means that either the flow rate needs to be maximized by increasing the velocity of the airflow or the average pressure on the vacuum area should be maximized. The design formulation can be defined as follows.

$$
\begin{array}{ll}
\text { Find } & X_{1}, X_{2}, X_{3}, X_{4} \\
\text { to maximize } & E=\frac{P_{\text {air }}}{P_{\text {shaft }}} \\
\text { subject to } & P_{\text {shaft }} \geq 350 \mathrm{~W} \\
& X_{L} \leq X_{i} \leq X_{U} \text { for } i=1 \text { to } 4
\end{array}
$$

where $P_{\text {air }}=P_{s} Q$ and $P_{\text {shaft }}=\frac{2 \pi N T}{60}$.

Table 2. DOE points and analysis results

\begin{tabular}{cccccccc}
\hline Case & $X_{1}$ & $X_{2}$ & $X_{3}$ & $X_{4}$ & $\mathrm{Ps}[\mathrm{kPa}]$ & $\mathrm{Q}[\mathrm{l} / \mathrm{s}]$ & $\mathrm{E}[\%]$ \\
\hline 1 & 0 & + & - & - & 13.78 & 11.75 & 55.1 \\
\hline 2 & - & - & - & - & 13.63 & 10.75 & 52.8 \\
\hline 3 & - & - & - & + & 13.43 & 10.56 & 51.3 \\
\hline 4 & - & - & + & - & 13.39 & 10.69 & 53.2 \\
\hline 5 & - & - & + & + & 13.50 & 10.24 & 43.9 \\
\hline 6 & - & + & - & - & 13.39 & 10.21 & 50.9 \\
\hline 7 & - & + & - & + & 14.27 & 12.16 & 56.9 \\
\hline 8 & - & + & + & - & 13.46 & 10.47 & 52.3 \\
\hline 9 & - & + & + & + & 13.48 & 11.78 & 51.9 \\
\hline 10 & + & - & - & - & 13.49 & 11.82 & 52.3 \\
\hline 11 & + & - & - & + & 13.19 & 11.88 & 52.5 \\
\hline 12 & + & - & + & - & 13.29 & 11.98 & 52.1 \\
\hline 13 & + & - & + & + & 13.43 & 11.66 & 51.3 \\
\hline 14 & + & + & - & - & 13.66 & 11.17 & 53.9 \\
\hline 15 & + & + & - & + & 12.94 & 11.66 & 54.7 \\
\hline 16 & + & + & - & - & 11.87 & 11.17 & 43.7 \\
\hline 17 & + & + & + & + & 12.98 & 11.68 & 49.5 \\
\hline 18 & - & 0 & + & 0 & 11.49 & 10.99 & 43.4 \\
\hline 19 & + & 0 & 0 & 0 & 14.07 & 12.11 & 56.8 \\
\hline 20 & 0 & - & 0 & 0 & 14.01 & 12.07 & 55.9 \\
\hline 21 & 0 & + & 0 & 0 & 12.78 & 11.61 & 54.1 \\
\hline 22 & 0 & 0 & - & 0 & 13.65 & 10.93 & 49.1 \\
\hline 23 & 0 & 0 & + & 0 & 13.94 & 10.76 & 46.5 \\
\hline 24 & 0 & 0 & 0 & - & 13.71 & 11.56 & 54.8 \\
\hline 25 & 0 & 0 & 0 & + & 13.85 & 11.84 & 54.1 \\
\hline Initial & 0 & 0 & 0 & 0 & 12.49 & 11.65 & 52.7 \\
\hline & & & & & & &
\end{tabular}

Since the flow path efficiency cannot be explicitly obtained, the DOE was used for the design optimization. The face-centred central composite design (FCCD) method, which is a three-level central composite planning method, was used for the DOE. Because the FCCD only generates DOE points within the design bounds, it is the only applicable DOE method because the optimum values of the design variables need to be within the design bounds. The lower bound, midpoint, and upper bounds of the four 
design variables for the FCCD are the same as those in Table 1, and the 25 design points for the FCCD and their analysis results are presented in the next section. The lower bound, middle, and upper bound are expressed as "_", "0", and "+", respectively.

\subsection{Optimization Results}

From the results of the CFD analysis, the flow path efficiency for Case 7 shows the best result (56.9\%), which improves the flow path efficiency by a difference of approximately $4.2 \%$ compared to the initial model $(52.7 \%)$ as shown in Table 2. In fact, the initial model used in this study was verified and improved through several analyses within the company, such that the current efficiency is still acceptable for mass production. However, a parametric study and design optimization had never been performed for the initial model; thus, the company expected better results through the parametric study and design optimization.

Through the design optimization in this study, the flow path efficiency for the initial model was improved by $4.2 \%$, which is difficult to achieve in reality because the design limitations are quite severe. Nevertheless, the shape change of the fan model increases the flow rate and the averaged pressure on the vacuum region, which lead to increase the flow path efficiency. The CFD results of the initial model and optimum model are compared in Figs. 11 through 13 in more detail.

Fig. 11 shows the velocity streamlines for the initial and optimum models when the diameter of the orifice is $13 \mathrm{~mm}$, which is currently used in the product of the company. As shown in Table 2, the airflow rate of the optimum model is higher than that of the initial model. Accordingly, the velocity streamlines for the optimum model pass through the flow path more smoothly than those for the initial model, as shown in Fig. 11. In addition, the velocity streams for the optimum model are less tangled because the vortices occur less frequently in the optimum model than in the initial model.

Similar results are observed in the plots of the velocity vectors, which are shown in the cross-section of the fan motor unit (Fig. 12). The velocity vectors for the optimum model are longer and denser than those for the initial model, especially in the vicinity of the impeller where the high velocities occurred. The air passing through the diffuser also flows out to the outlet with a higher speed in the optimum model than in the original model. Likewise, the pressure of the optimum model at the rotary part of the fan motor unit is lower than the initial model, which indicates

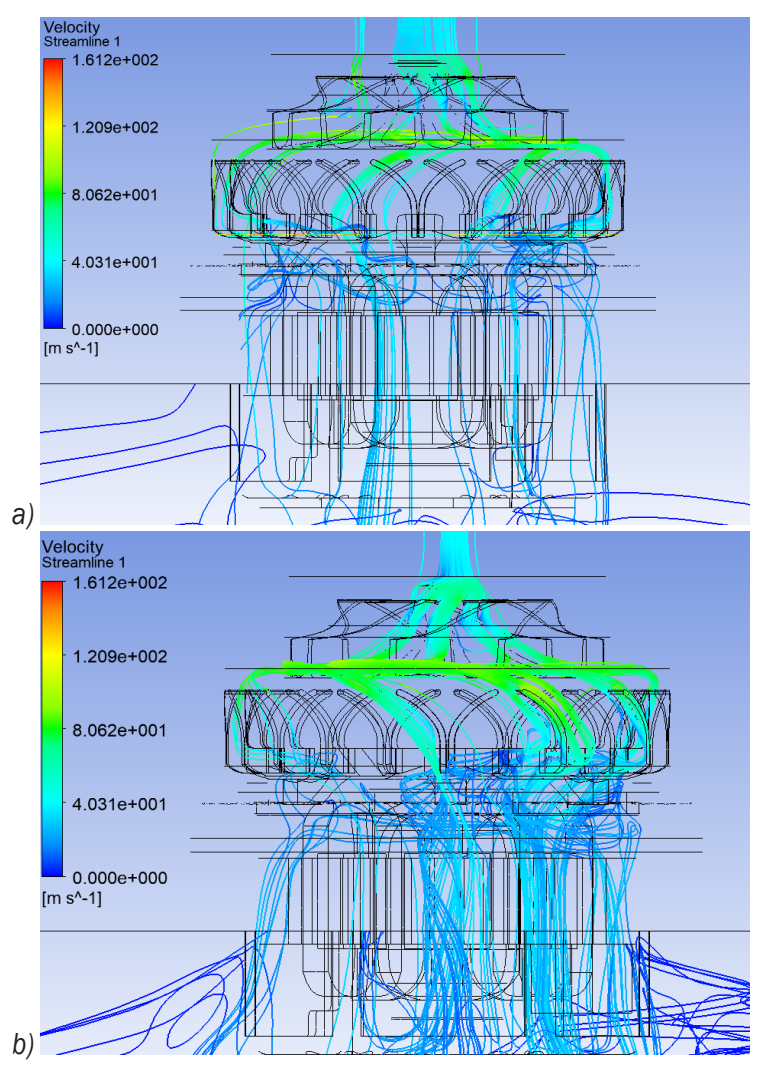

Fig. 11. Velocity streamlines; a) initial model, and b) optimum model

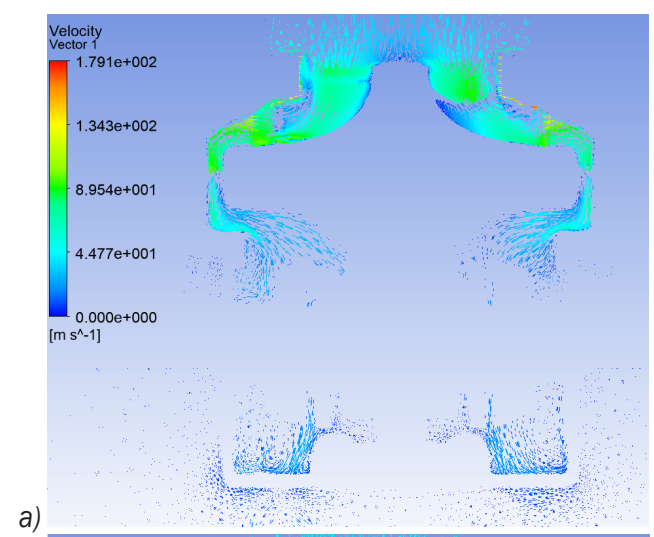

a)

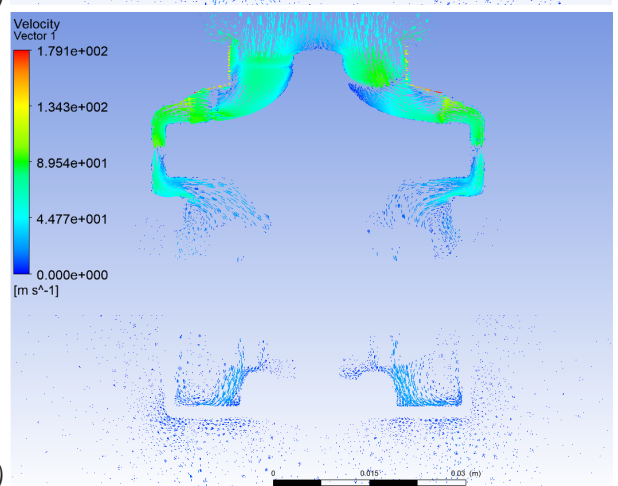

Fig. 12. Velocity vectors; a) initial model, and b) optimum model 


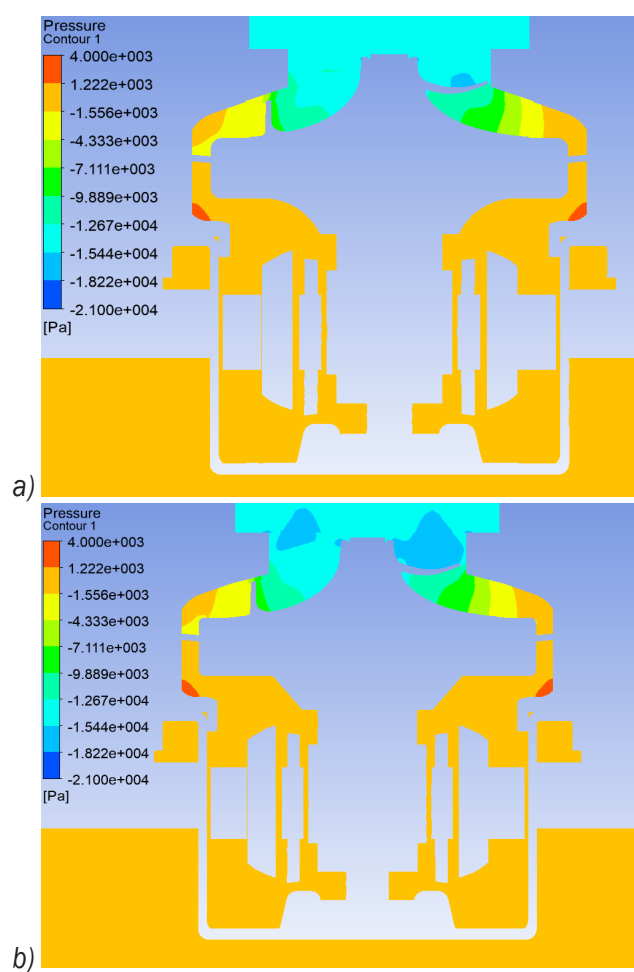

Fig. 13. Pressure contours inside the fan motor unit; a) initial model, and b) optimum model

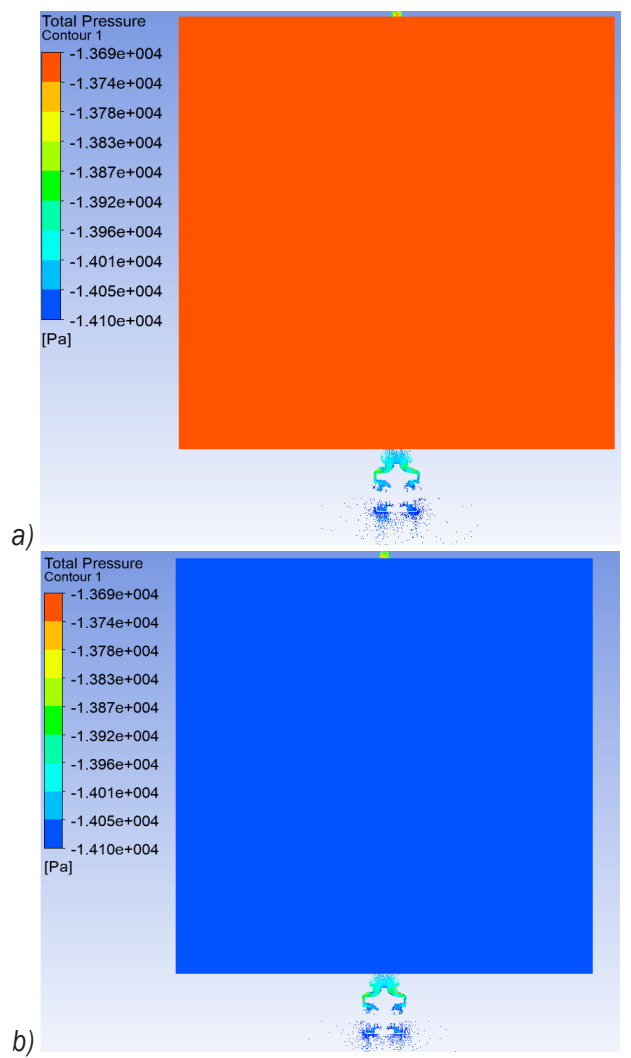

Fig. 14. Pressure contours in the vacuum region; a) initial model, and b) optimum model that the optimum model better intakes the air than the initial model as shown in Fig. 14.

Fig. 14 shows the pressure contours obtained for the initial and optimum models. The difference between the maximum and minimum pressure in Fig. 14 is less than $3 \mathrm{~Pa}$, which is much smaller than their absolute values, so that the values of the pressure are shown to be the same. However, the average pressure on the vacuum region in the optimum model is higher than the one in the initial model, which yields the higher flow path efficiency than the initial model.

According to Bernoulli's principle, as the airflow velocity of the optimum model increases, the pressure on the entire wall of the vacuum region decreases, which increases the absolute value of the pressure; thus, the flow path efficiency also increases proportionally with the increase in the absolute value of the pressure. The pressure drop is clearly shown near the high-velocity regions, i.e. the inlets of the orifice and the fan motor unit.

Figs. 15 to 17 show the flow rate, suction power, and flow path efficiency, respectively, obtained for the initial and optimum models. As the rotation speed increases, the air is rapidly drawn into the fan motor unit, and thus, the airflow rate also linearly increases by the affinity laws of turbo-machines [20] as shown in Fig. 15. Likewise, because the suction power is proportional to the cube of the rotation speed by the affinity laws, it also rapidly increases according to the rotation speed as shown in Fig. 16. However, as the flow power does not increase as much as the suction power because of the energy loss in the fast airflow, the rate of increases in the flow path efficiency gradually decreases. Nevertheless, the optimum model is $2 \%$ to $2.5 \%$ more efficient than the initial model, especially in the high-rotation speed region.

It can be said that $4.2 \%$ improvement is not significant, and it might include the error caused by an idealized geometry, FE models, and CFD analysis. The real fan model might not achieve the same efficiency as the analysis results do. The experimental results were not shown in this study owing to the confidential reason of the company; they are similar with the CFD results within $5 \%$ of error. The experimental results on the airflow rates and the suction powers were shown to be up to $2 \%$ to $3 \%$ lower than the analysis results especially at the high rotational speeds due to the idealization of the analytical models and nonlinear flow phenomena. In addition, the experimental results for the flow path efficiency were also $5 \%$ lower than the analysis results because the experimental results included efficiency of the electromagnetic cores, motors, and inverters. 
Nevertheless, the optimum model still shows consistently better results than the initial model for various rotational speeds and similar results with the experimental results. Thus, it can be confirmed that the analysis results are enough to represent the experimental results and the optimum model is indeed superior to the original model.

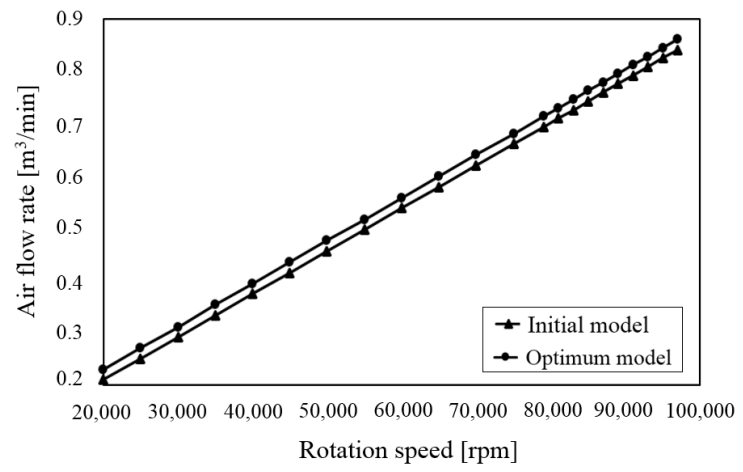

Fig. 15. Airflow rate for various fan rotation speeds

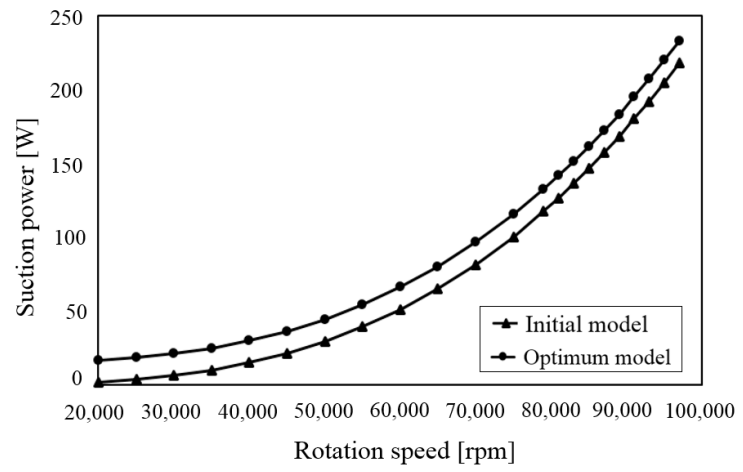

Fig. 16. Suction power for various fan rotation speeds

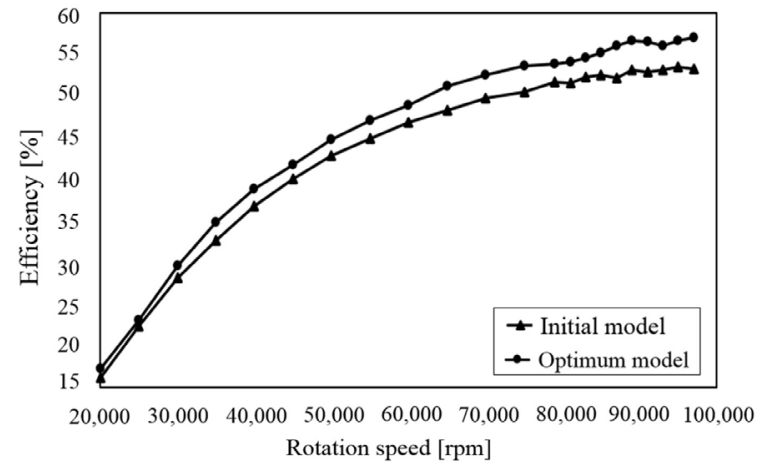

Fig. 17. Efficiency for various fan rotation speeds

\section{DISCUSSION}

In previous research, there were no such cases of modelling and analysing the entire flow field outside the fan motor. In this study, the flow around the fan motor unit, the fan, and the flow path were analysed in the same condition as the actual experimental environment.

It is difficult to change the shape of the motor and the surrounding electrical equipment. The fan, which has been used in the company, is already optimized, and the lower body of the fan motor unit hardly affects the flow path efficiency, so that the shape of the diffuser vane is only selected as a design target. The parametric studies of six shape variables related to the diffuser vane using Plackett Burman design confirmed that the diffuser diameter, number of guide vanes, diffuser thickness, and the curvature radius of the diffuser are the main factors affecting the flow path efficiency.

Since the velocity and direction of the air flow change rapidly when it enters the diffuser from the impeller, the associated four variables are the most important factors affecting the flow path efficiency. In contrast, the guide vane only guides the air flow downward, the associated two variables, the curvature radius of the vane and the vane width, do not have a significant effect on the flow path efficiency.

Using the four design variables, the objective of the optimum design is to maximize the ratio of the input power to the fluid power, which is the multiplication of the average pressure over the vacuum region and flow rate. The optimization was performed using FCCD, and the optimum model increased the flow path efficiency by $4.2 \%$ in comparison to the initial model.

Comparing the velocity streamlines and vectors of the optimum model and initial model, the air flow of the optimum model smoothly passes through the flow path at high speed and produces less vortex than that of the initial model. Furthermore, the pressure contour figure shows that the average pressure in the vacuum region of the optimum model is higher than that of the initial model, which can increase the flow path efficiency.

The performances of the optimum model, flow rate, suction power, and flow efficiency, are verified by varying the rotation speed with $20,000 \mathrm{rpm}$ to $100,000 \mathrm{rpm}$. As a result, it is confirmed that the optimum model is always superior to the initial model for all cases.

\section{CONCLUSIONS}

In this study, the diffuser in the fan motor unit for a compact vacuum cleaner was optimized to improve the flow path efficiency of the initial model. For this, CFD analysis and DOE were performed, and the optimum model was obtained so that it maximizes the 
flow path efficiency subject to the constraints for the given suction power. The conclusions of the study are as follows.

- The CFD analysis was performed on the entire flow field of the fan motor unit for a compact vacuum cleaner.

- Through parametric studies, the diameter of the diffuser, the number of guide vanes, the thickness of the diffuser, and the radius of the diffuser curvature were selected as the main design variables affecting the flow path efficiency of the fan motor unit.

- The optimum model using the DOE increased the flow path efficiency by $4.2 \%$, in comparison to the initial model, by increasing the airflow rate and wall pressure in the vacuum region.

- The optimum model yields better analysis results than the initial model for flow rate, suction power, and flow path efficiency for all applicable rotation speeds.

This study was carried out to improve the performance of the original fan motor unit of the vacuum cleaner currently being developed by LG electronics. Further studies will be performed to minimize its weight (by changing the dimensions of the motor housing) and to reduce noise and heat generation occurring at the motor unit for the mass production of the fan motor unit.

\section{ACKNOWLEDGEMENTS}

The research described in this paper was supported by LG Electronics and the Human Resources Development program (No. 20164030201230) of the Korea Institute of Energy Technology Evaluation and Planning (KETEP) grant funded by the Ministry of Trade, Industry and Energy. This support is greatly appreciated.

\section{REFERENCES}

[1] Lee, K.C., (2002). Flow Analysis of a Low-Noise Turbo-Fan for a Vacuum Cleaner, MSc. D. Thesis, Seogang University, Seoul.

[2] Park, J.W. (2008). The Performance Improvement and Optimum Design of Vacuum Cleaner by Analysis of the Flow Around Centrifugal Fan. MSc. Thesis, Chonam University, Gwangju.

[3] Kim, J.Y., Kwac, L.K., An, J.S., Yang, D.J., Song, K.S., Park, K.H. (2004). The flow analysis for vibration and noise diagnostic of vacuum cleaner fan motor. Transactions of the Korean Society of Machine Tool Engineers, vol. 13, no. 4, p. 147-163.

[4] Kale, P.P., Patil, M.B., Korade, D.N., Jagtap, K.R. (2016). Experimental and CFD analysis of vacuum cleaner exhaust muffler. Journal of Mechanical and Civil Engineering, vol. 13, no. 1, p. 21-27, DOI:10.9790/1684-13122127.

[5] Rihtaršič, J., Šubbelj, M., Hočevar, M., Duhovnik, J. (2008). Flow analysis through the centrifugal impeller of a vacuum cleaner unit. Strojniški vestnik - Journal of Mechanical Engineering, vol. 54, no. 2, p. 81-93.

[6] Li, H. (2009). Fluid flow analysis of a single-stage centrifugal fan with a ported diffuser. Engineering Applications of Computational Fluid Mechanics, vol. 3, no. 2, p. 147-163, DOI:10.1080/19942060.2009.11015261.

[7] Park, C., Jun, S., Park, K., Lee, S., Chang, K. (2016). Methodology for system-level analysis of a fan-motor design for a vacuum cleaner. Proceeding of the Institute of Mechanical Engineers, Part C: Journal of Mechanical Engineering Science, vol. 231, no. 20, p. 3840-3854, DOI:10.1177/0954406216651650.

[8] Lee, M.J. (2009). Aerodynamic Design of 3-D Turbo Fan. MSc Thesis, Inha University, Incheon.

[9] Kim, J.-H., Ovgor, B., Cha, K.-H., Kim J.-H., Lee, S., Kim, K.-Y. (2014). Optimization of the aerodynamic and aeroacoustic performance of an axial-flow fan. AIAA Journal, vol. 52, no. 9, p. 2032-2044, DOl:10.2514/1.J052754.

[10] PIAnO manual (2015). PIDOTEC Inc., Seoul.

[11] ANSYS Fluent 16.0 theory guide (2016). from https://uiuc-cse. github.io/me498cm-fa15/lessons/fluent/refs/ANSYS\%20 Fluent\%20Theory\%20Guide.pdf, assessed on 2016-07-01.

[12] ANSYS 16.0 CFD Tutorials (2016). from http://148.204.81.206/Ansys/150/ANSYS\%20Fluent\%20 Tutorial\%20Guide.pdf, assessed on 2016-07-01.

[13] Launder, B.E., Spalding, D.B. (1974). The numerical computation of turbulent flows. Computer Methods in Applied Mechanics and Engineering, vol. 3, no. 2, p. 269-289, DOl:10.1016/0045-7825(74)90029-2.

[14] Bardina, J.E., Huang, P.G., Coakley, T. (1997) Turbulence modeling validation. 28th Fluid Dynamics Conference, DOI:10.2514/6.1997-2121.

[15] Menter, F.R. (1994). Two-equation eddy-viscosity turbulence models for engineering applications, AIAA Journal, vol. 32, no. 8, p. 1598-1605, Dol:10.2514/3.12149.

[16] Menter, F.R., Kuntz, M., Langtry, R. (2003). Ten years of industrial experience with the SST turbulence model. Turbulence, Heat and Mass Transfer, vol. 4, p. 625-632.

[17] Simoes, M.R., Montojos, B.G., Moura, N.T., Su, J, (2009). Validation of turbulence model for simulation of axial flow compressor. Proceedings of 20th International Congress of Mechanical Engineering, Gramado.

[18] Hillewaert, K., Van den Braembussche, R.A. (1999). Numerical simulation of impeller-volute interaction in centrifugal compressors. Journal of Turbomachinery, vol. 121, no. 3, p. 603-607, DOI:10.1115/1.2841358.

[19] Kapun, A., Čurkovič, M., Hace, A., Jezernik, K. (2008). Identifying dynamic model parameters of a BLDC motor. Simulation Modeling Practice and Theory, vol. 16, no. 9, p. 1254-1265, D0l:10.1016/j.simpat.2008.06.003.

[20] Brennen, C.E. (2011). Hydrodynamics of Pumps, Cambridge University Press, New York, DOI:10.1017/CB09780511976728. 